\title{
SINGULAR YAMABE METRICS AND INITIAL DATA WITH EXACTLY KOTTLER-SCHWARZSCHILD-DE SITTER ENDS II. GENERIC METRICS
}

\author{
Piotr T. Chruściel, Frank Pacard, and Daniel Pollack
}

\begin{abstract}
We present a gluing construction which adds, via a localized deformation, exactly Delaunay ends to generic metrics with constant positive scalar curvature. This provides time-symmetric initial data sets for the vacuum Einstein equations with positive cosmological constant with exactly Kottler-Schwarzschild-de Sitter ends, extending the results in [5].
\end{abstract}

\section{Introduction}

There exists very strong evidence suggesting that we live in a world with strictly positive cosmological constant $\Lambda[10,9]$. This leads to a need for a better understanding of the space of solutions of Einstein equations with $\Lambda>0$. In [5] it has been shown how time-symmetric initial data for such space-times, which contain asymptotically Delaunay ends, can be deformed to initial data with exactly Delaunay ends. The resulting space-times contain regions of infinite extent on which the metric takes exactly the Kottler-Schwarzschild-de Sitter form. Moreover, such ends can be used for creating wormholes, or connecting initial data sets, provided the neck parameters of the ends match. The object of this note is to show that generic constant positive scalar curvature metrics can be deformed, by a local deformation, to constant positive scalar curvature metrics containing asymptotically Delaunay ends. The method of [5] is then used to obtain exactly Delaunay ends.

We further point out that the neck parameter of each new asymptotically Delaunay end can be arbitrarily prescribed within an interval $\left(0, \epsilon_{0}\right)$, for some $\epsilon_{0}>0$. This flexibility in prescribing the neck sizes guarantees that the exactly Delaunay ends can be matched to perform gluings.

\section{Statement of the result}

We begin with some terminology. A static KID is a function $N$ satisfying

$$
D_{i} D_{j} N-N R_{i j}-\Delta_{g} N g_{i j}=0 .
$$

We shall say that there exist no local static KIDs near $p$ if there exist sequences $0<\eta_{i}<\delta_{i} \rightarrow_{i \rightarrow \infty} 0$ such that there exist no nontrivial solution of (1) on the annuli

$$
A_{p}\left(\eta_{i}, \delta_{i}\right):=\bar{B}_{p}\left(\delta_{i}\right) \backslash B_{p}\left(\eta_{i}\right),
$$

Received by the editors March 19, 2008. 
where $B_{p}(r)$ is a geodesic ball centered at $p$ of radius $r$. Observe that (1) forms a set of overdetermined equations, it is natural to expect that for generic metrics on $M$ there will not be any local static KIDs at any point $p \in M$. Precise statements to this effect have been proved in [1], both for unconstrained metrics, and for metrics with constant non-positive scalar curvature.

It is well known that there exists a one parameter family of constant positive scalar curvature conformal metrics on $\mathbb{R} \times S^{n-1}$. In the literature, these metrics are usually referred to as Kottler-Schwarzschild-de Sitter metrics [5] or Delaunay metrics [8] or even Fowler metrics [6]. This family of metrics is parameterized by a parameter which is called the "neck size". Let us describe these briefly since they are at the heart of our result. We assume $n \geq 3$ and we set

$$
\epsilon_{*}:=\left(\frac{n-2}{n}\right)^{\frac{4}{n-2}} .
$$

For all $\epsilon \in\left(0, \epsilon_{*}\right]$, we define $v_{\epsilon}$ to be the unique solution of

$$
\partial_{t}^{2} v_{\epsilon}-\left(\frac{n-2}{2}\right)^{2} v_{\epsilon}+\frac{n(n-2)}{4} v_{\epsilon}^{\frac{n+2}{n-2}}=0
$$

with $v_{\epsilon}=\epsilon$ and $\partial_{t} v_{\epsilon}(0)=0$. The parameter $\epsilon$ is called the neck size of the associated Delaunay metric

$$
g_{\epsilon}=u_{\epsilon}^{\frac{4}{n-2}} d x^{2}=v_{\epsilon}^{\frac{4}{n-2}}\left(d t^{2}+g_{S^{n-1}}\right)
$$

where

$$
u_{\epsilon}(x):=|x|^{\frac{2-n}{2}} v_{\epsilon}(-\log |x|),
$$

and where $d x^{2}$ denotes the Euclidean metric in $\mathbb{R}^{n}$ and $g_{S^{n-1}}$ denotes the canonical metric on $S^{n-1}$. The metric $g_{\epsilon}$ has constant scalar curvature equal to $R \equiv n(n-1)$. Observe that, without loss of generality, we can normalize the metrics we are interested in to have (constant) scalar curvature equal to $n(n-1)$.

Building on the results in $[2,4,5]$, and using a simple perturbation argument, we prove the:

THEOREM 2.1. Let $(M, g)$ be a smooth $n$-dimensional Riemannian manifold with constant positive scalar curvature $R \equiv n(n-1), n \geq 3$. Let $p \in M$ and suppose that there exist no local static KIDs near $p$. Then for any $\rho>0$ there exists $\epsilon_{0}>0$ such that for any $\epsilon \in\left(0, \epsilon_{0}\right]$ there exists a smooth metric with constant positive scalar curvature $R \equiv n(n-1)$ which coincides with $g$ in $M \backslash B_{p}(\rho)$ and which coincides with a Delaunay metric $g_{\epsilon}$ with neck size parameter $\epsilon$ in a punctured ball centered at $p$.

As already pointed out, a generic metric will satisfy the "no local static KIDs" property at every point. Since both our construction and that of [5] are purely local, the construction can be repeated at any chosen finite, or countably infinite, collection of points $p_{i} \in M$ to produce complete constant positive scalar curvature metrics with a countable number of exactly Delaunay ends.

Remark: It would be of interest to generalise this construction to general, not necessarily time-symmetric, general relativistic initial data sets $(M, g, K)$. 
2.1. Proof of Theorem 2.1: We choose $p \in M$ and $\rho>0$. There exists $\bar{\rho} \leq \rho$ such that for all $\delta<\bar{\rho}$ the linear operator ${ }^{1}$

$$
L:=\Delta_{g}+n
$$

is injective on $\mathcal{C}^{2}\left(\bar{B}_{p}(\delta)\right)$, the space of $\mathcal{C}^{2}$-functions which are defined on $\bar{B}_{p}(\delta)$ and which vanish on the boundary of this domain. The injectivity follows easily from the fact that in geodesic normal coordinates the metric $g$ can be expanded as $g_{i j}=$ $\delta_{i}^{j}+\mathcal{O}\left(|x|^{2}\right)$ and hence, for all $\delta>0$ small enough, we can estimate

$$
\int_{\bar{B}_{p}(\delta)}\left(\frac{1}{2}\left|\nabla_{\dot{g}} v\right|^{2}-2 n v^{2}\right) d v_{\dot{g}} \leq \int_{\bar{B}_{p}(\delta)}\left(\left|\nabla_{g} v\right|^{2}-n v^{2}\right) d v_{g}
$$

where $\stackrel{g}{g}$ denotes the Euclidean metric. But, if $\lambda_{1}$ is the first eigenvalue of $-\Delta_{\grave{g}}$ on the unit ball of $\mathbb{R}^{n}$, we have

$$
\lambda_{1} \int_{\bar{B}_{p}(\delta)} v^{2} d v_{\grave{g}} \leq \delta^{2} \int_{\bar{B}_{p}(\delta)}\left|\nabla_{\grave{g}} v\right|^{2} d v_{\grave{g}}
$$

and we conclude that

$$
0 \leq \int_{\bar{B}_{p}(\delta)}\left(\left|\nabla_{g} v\right|^{2}-n v^{2}\right) d v_{g}
$$

for all $\delta$ small enough. This clearly implies that $L$ is injective. Since we have assumed that there are no local static KIDs near $p$, by the arguments in [1] there exists $0<$ $\eta<\delta<\bar{\rho}$ such that there are no static KIDs on the annulus

$$
A_{p}(\eta, \delta)=\bar{B}_{p}(\delta) \backslash B_{p}(\eta) .
$$

Therefore, we conclude that we can choose $0<\eta<\delta \leq \rho$ such that there are no static KIDs on the annulus $A_{p}(\eta, \delta)$ and the operator $L$ is injective on $\mathcal{C}^{2}\left(\bar{B}_{p}(\delta)\right)$.

In $\bar{B}_{p}(\delta)$, we now deform the metric $g$ to a family of metrics which are conformally flat in a small neighborhood $p$ and which still have constant scalar curvature equal to $R=n(n-1)$ in $\bar{B}_{p}(\delta)$. The new metrics will match continuously the original ones along the boundary $\partial B_{p}(\delta)$, but in general their derivatives will not agree there.

LEMma 2.2. There exists $0<r_{0} \leq \eta$ and a family of smooth constant scalar curvature metrics $g^{r}$, with $r \in\left(0, r_{0}\right]$, which are defined in $\bar{B}_{p}(\delta)$, have constant scalar curvature $R \equiv n(n-1)$, are conformally flat in $\bar{B}_{p}(r)$, the ball of radius $r$ centered at $p$ (radius computed with respect to the metric $g$ ) and for which

$$
L_{r}:=\Delta_{g^{r}}+n
$$

is injective on $\mathcal{C}^{2}\left(\bar{B}_{p}(\delta)\right)$. Moreover

$$
\left\|g^{r}-g\right\|_{L^{\infty}\left(B_{p}(\delta)\right)}+r\left\|\nabla_{g}\left(g^{r}-g\right)\right\|_{L^{\infty}\left(B_{p}(\delta)\right)} \leq c r^{\gamma},
$$

for any $\gamma<2$, for some constant $c=c(\gamma)$. Furthermore, for any $k \in \mathbb{N}$, the sequence of metrics $g^{r}$ converges to $g$ in $\mathcal{C}^{k}$-topology, on compacts of $\bar{B}_{p}(\delta) \backslash\{p\}$, as $r$ tends to zero.

\footnotetext{
${ }^{1}$ Observe that our Laplacian is the sum of second derivatives.
} 
Proof. We agree that the geodesic balls, the gradient and norm are taken with respect to the metric $g$. We consider geodesic normal coordinates $x:=\left(x_{1}, \ldots, x_{n}\right)$ near $p$. As already mentioned, in these coordinates, the metric $g$ can be expanded as

$$
g_{i j}=\delta_{i}^{j}+\mathcal{O}\left(|x|^{2}\right) .
$$

We choose a cutoff function $\chi$ which is identically equal to 1 in the unit ball of $\mathbb{R}^{n}$ and identically equal to 0 outside the ball of radius 2 . Given $r \in(0, \delta / 2)$, we consider a metric $\bar{g}^{r}$ whose coefficients near $p$ are given by

$$
\bar{g}_{i j}^{r}:=\chi(\cdot / r) \delta_{i}^{j}+(1-\chi(\cdot / r)) g_{i j} .
$$

Observe that

$$
\left\|\bar{g}^{r}-g\right\|_{L^{\infty}}+r\left\|\nabla_{g}\left(\bar{g}^{r}-g\right)\right\|_{L^{\infty}}+r^{2}\left\|\nabla_{g}^{2}\left(\bar{g}^{r}-g\right)\right\|_{L^{\infty}} \leq c r^{2} .
$$

for some constant $c>0$ which does not depend on $r \leq \delta / 2$.

Let us denote by $\bar{R}_{r}$ the scalar curvature of the metric $\bar{g}^{r}$ and recall that $R=$ $n(n-1)$ is the scalar curvature of the metric $g$. We have $\bar{R}_{r}=R$ in $\bar{B}_{p}(\delta) \backslash B_{p}(2 r)$ and $\bar{R}_{r}=0$ in $\bar{B}_{p}(r)$. Finally, in the annulus $\bar{B}_{p}(2 r) \backslash B_{p}(r)$, we only have the estimate

$$
\left\|\bar{R}_{r}\right\|_{L^{\infty}} \leq c
$$

for some constant $c>0$ independent of $r \leq \delta / 2$. This follows at once from the fact that the expression of the scalar curvature in terms of the coefficients of the metric involves the coefficients of the metric and their derivatives up to order 2 which, thanks to (5), are bounded independently of $r \leq \delta / 2$.

Now, we explain how to solve the equation

$$
\Delta_{\bar{g}^{r}} u-\frac{n-2}{4(n-1)} \bar{R}_{r} u+\frac{n(n-2)}{4} u^{\frac{n+2}{n-2}}=0,
$$

for $r$ small enough. Once this equation is solved and assuming that the function $u>0$, the metric

$$
g^{r}:=u^{\frac{4}{n-2}} \bar{g}^{r},
$$

will be a constant scalar curvature metric equal to $R \equiv n(n-1)$ defined in $\bar{B}_{p}(\delta)$.

We set $u=1+v$ and rewrite the above equation as

$$
L v=\left(\Delta_{g}-\Delta_{\bar{g}^{r}}\right) v+\frac{n-2}{4(n-1)}\left(\bar{R}_{r}-R\right)(1+v)-\frac{n(n-2)}{4}\left((1+v)^{\frac{n+2}{n-2}}-1-\frac{n+2}{n-2} v\right) .
$$

For short, we denote by $N_{r}(v)$ the right hand side of this equation and we assume that the function $v$ is small enough, say $\|v\|_{L^{\infty}} \leq 1 / 2$, so that $1+v>0$.

We fix $\ell>n / 2$. Using the fact that $\bar{R}_{r}-R$ is supported in $B_{p}(2 r)$ and is bounded in this set, it is easy to check that there exists a constant $c>0$ (which does not depend on $r \in(0, \delta / 2))$ such that

$$
\left\|\left(\bar{R}_{r}-R\right)(1+v)\right\|_{L^{\ell}} \leq c\left(1+\|v\|_{L^{\infty}}\right) r^{n / \ell},
$$

and

Similarly,

$$
\left\|\left(\bar{R}_{r}-R\right)\left(v-v^{\prime}\right)\right\|_{L^{\ell}} \leq c r^{n / \ell}\left\|v-v^{\prime}\right\|_{L^{\infty}} .
$$

$$
\left\|(1+v)^{\frac{n+2}{n-2}}-1-\frac{n+2}{n-2} v\right\|_{L^{\ell}} \leq c\|v\|_{L^{\infty}}^{2},
$$


and

$$
\left\|(1+v)^{\frac{n+2}{n-2}}-\left(1+v^{\prime}\right)^{\frac{n+2}{n-2}}-\frac{n+2}{n-2}\left(v-v^{\prime}\right)\right\|_{L^{\ell}} \leq c\left(\|v\|_{L^{\infty}}+\left\|v^{\prime}\right\|_{L^{\infty}}\right)\left\|v-v^{\prime}\right\|_{L^{\infty}},
$$

provided $\|v\|_{L^{\infty}} \leq 1 / 2$ and $\left\|v^{\prime}\right\|_{L^{\infty}} \leq 1 / 2$

Thanks to (5) we also check that

$$
\left\|\left(\Delta_{g}-\Delta_{\bar{g}^{r}}\right) v\right\|_{L^{\ell}} \leq \operatorname{cr}\|v\|_{W^{2, \ell}} .
$$

with perhaps another constant $c>0$ which again does not depend on $r \in(0, \delta / 2)$. Indeed, the difference between these two operators involves a second order differential operator whose coefficients can be estimated by the difference of the coefficients of the two metrics and a first order differential operator whose coefficients can be estimated by the gradient of the difference between the coefficients of the two metrics.

By hypothesis, the operator

$$
L:=\Delta_{g}+n,
$$

is an isomorphism from $\stackrel{\circ}{W}^{2, \ell}\left(B_{p}(\delta)\right)$ into $L^{\ell}\left(B_{p}(\delta)\right)$ (Recall that $\stackrel{\circ}{W}^{2, \ell}\left(B_{p}(\delta)\right.$ ) is the completion of the space of smooth functions on $B_{p}(\delta)$ which vanish on $\partial B_{p}(\delta)$ with

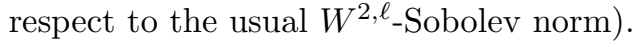

Collecting these we find that

$$
\left\|L^{-1} N_{r}(v)\right\|_{W^{2, \ell}} \leq c\left(r^{n / \ell}+\left(r+r^{n / \ell}\right)\|v\|_{L^{\infty}}+\|v\|_{L^{\infty}}^{2}\right),
$$

and

$$
\left\|L^{-1}\left(N_{r}(v)-N_{r}\left(v^{\prime}\right)\right)\right\|_{W^{2, \ell}} \leq c\left(r+r^{n / \ell}+\|v\|_{L^{\infty}}+\left\|v^{\prime}\right\|_{L^{\infty}}\right)\left\|v-v^{\prime}\right\|_{L^{\infty}} .
$$

Using the embedding $W^{2, \ell}\left(B_{p}(\delta)\right) \longrightarrow L^{\infty}\left(B_{p}(\delta)\right)$, we conclude, from the fixed point theorem for contraction mappings, that the nonlinear operator

$$
v \mapsto L^{-1} N_{r}(v)
$$

has a (unique) fixed point $v_{r}$ in the ball of radius $2 c r^{n / \ell}$ in $W^{2, \ell}\left(B_{p}(\delta)\right.$ ), provided $r>0$ is small enough, say $r \in\left(0, r_{0}\right]$. Note that the constant $c=c_{\ell}$ depends on $\ell$ since it depends on the norm of the Sobolev embedding. This completes the proof of the existence of a positive solution of (6). The fact that $v_{r}$ (and hence $u_{r}:=1+v_{r}$ ) is smooth follows from classical elliptic regularity.

The metric which appears in the statement of the result is

$$
g^{r}:=\left(1+v_{r}\right)^{\frac{4}{n-2}} \bar{g}^{r} .
$$

The first estimate in (4) follows from the construction itself and the embedding $W^{2, \ell}\left(B_{p}(\delta)\right) \longrightarrow L^{\infty}\left(B_{p}(\delta)\right)$ when $\ell>n / 2$, while the second estimate in (4) follows from the construction and the embedding $W^{2, \ell}\left(B_{p}(\delta)\right) \longrightarrow W^{1, \infty}\left(B_{p}(\delta)\right)$ when $\ell>n$.

Perhaps, it is now time to comment about the choice of $\ell$. Observe that functions if $W^{2, \ell}$ are in $L^{\infty}$ provided $\ell>n / 2$ while their derivatives are in $L^{\infty}$ provided $\ell>n$. In order to estimate the nonlinearities in the equation it was easy to work with bounded functions, this is the main reason why we have chosen $\ell>n / 2$. Now, in the statement of the result, we claim $L^{\infty}$ bounds both on the solution itself and its partial derivatives; to obtain those, it would have been tempting to work directly with $\ell>n$. However, with this latter choice we would have obtained the desired $L^{\infty}$ estimate for the partial 
derivatives of the solution, but we would not have obtained a good estimate for the $L^{\infty}$ norm of the solution itself; this is where $\ell>n / 2$ was necessary. Observe that, in principle, the solution of the fixed point problem does depend on $\ell$. However, if $\ell^{\prime}>\ell$ and $v_{r}^{\prime}$ and $v_{r}$ are the respective fixed points, it follows easily from (7) that $v_{r}^{\prime}=v_{r}$ for all $r$ small enough.

Finally, reducing $r_{0}$ if necessary, we claim that the operator

$$
\Delta_{g^{r}}+n
$$

is injective for all $r \in\left(0, r_{0}\right]$. To do so, simply use (5) which implies that the coefficients of $g^{r}-g$ and their derivatives are bounded in $L^{\infty}$-norm by a constant times $r^{\gamma-1}$. This implies that

$$
\left\|\left(\Delta_{g}-\Delta_{g^{r}}\right) v\right\|_{L^{\ell}} \leq c r^{\gamma-1}\|v\|_{W^{2, \ell}} .
$$

Finally, the claim then follows from a simple perturbation argument since $L$ is an isomorphism between $\stackrel{\circ}{W}^{2, \ell}\left(B_{p}(\delta)\right)$ and $L^{\ell}\left(B_{p}(\delta)\right)$ for any $\ell>1$.

The fact that, for any $k \in \mathbb{N}$, the sequence of metrics $g^{r}$ converges to $g$ in $\mathcal{C}^{k}$ topology, on compacts of $\bar{B}_{p}(\delta) \backslash\{p\}$, as $r$ tends to zero follows from elliptic regularity theory since, for all $r \in(0, \bar{r}]$, the function $v_{r}$ is a solution of

$$
\Delta_{g} v_{r}+n v_{r}+\frac{n(n-2)}{4}\left(\left(1+v_{r}\right)^{\frac{n+2}{n-2}}-1-\frac{n+2}{n-2} v_{r}\right)=0
$$

in $\bar{B}_{p}(\delta) \backslash B_{p}(\bar{r})$ which vanishes on $\partial B_{p}(\delta)$ and is bounded by a constant times $r^{\gamma}$ in $L^{\infty}$-norm.

The following result is essentially borrowed from [2]:

THEOREM 2.3. Let $\left(M_{0}, g_{0}\right)$ be a compact Riemannian manifold with smooth boundary $\partial M_{0}$. Assume that the scalar curvature is constant equal to $R=n(n-1)$, and that the operator

$$
\Delta_{g_{0}}+n
$$

acting on functions $\stackrel{\circ}{W}^{2, \ell}\left(M_{0}\right)$ is injective. Further assume that the metric $g_{0}$ is locally conformally flat in a neighborhood of a point $p \in M_{0}$. Then, there exists $\epsilon_{0}>0$ and for all $\epsilon \in\left(0, \epsilon_{0}\right]$ there exists a complete constant scalar curvature metric defined in $M_{0} \backslash\{p\}$ of the form

$$
\tilde{g}_{\epsilon}=e^{\phi_{\epsilon}} g_{0}
$$

where $\phi_{\epsilon}=0$ on $\partial M_{0}$. Furthermore, $\phi_{\epsilon}$ converges to 0 on compacts subsets of $M_{0} \backslash\{p\}$ in any $\mathcal{C}^{k}$-topology, for $k \in \mathbb{N}$.

Finally, in the neighborhood of $p$ where $g_{0}$ is conformally flat and where we can use coordinates $x \in \mathbb{R}^{n}$ with $x=0$ at $p$, the metric $\tilde{g}_{\epsilon}$ can be written as

$$
\tilde{g}_{\epsilon}=\tilde{u}_{\epsilon}^{\frac{4}{n-2}} d x^{2}
$$

where the function $u_{\epsilon}$ satisfies

$$
\tilde{u}_{\epsilon}(x)=|x|^{\frac{2-n}{2}} v_{\epsilon}\left(-\log |x|+t_{\epsilon}\right)(1+\mathcal{O}(|x|)),
$$

for some $t_{\epsilon} \in \mathbb{R}$, where the error term $\mathcal{O}(|x|)$ depends on $\epsilon$. 
REMARK 2.4. In addition, reducing $\epsilon_{0}$ if this is necessary, the solutions constructed are "unmarked nondegenerate" (see section 6 of [2] or Proposition 10 in [7] for a proof). We refer to [8] for the definition of unmarked nondegeneracy. In particular this implies that one can use $p \in M_{0}$ and $\epsilon$ as coordinates on the unmarked moduli space.

Proof. Existence follows from Theorem 1.1 in [2]. The fact that the construction is possible for any small value of the parameter $\epsilon$ is not explicit in the statement of Theorem 1.1 in [2] but it is implicit in the proof (see bottom of page 1184). The expansion of the metric close to $p$ follows directly from the construction in [2] but also follows from general results such as [3] and [6] (see (9) page 241 and Theorem 1 page 235 of $[6])$.

Finally, we recall the result of [4, Theorem 5.9]: Choose some $k>n / 2+4$. If there are no static KIDs on the annulus $A_{p}(\eta, \delta)$, then there exists $\zeta>0$ such that if $\tilde{g}$ is a constant scalar curvature metric metric on the annulus $\bar{B}_{p}(\delta) \backslash B_{p}(\eta / 2)$ satisfying

$$
\|g-\tilde{g}\|_{\mathcal{C}^{k}\left(A_{p}(\eta, \delta)\right)} \leq \zeta
$$

(recall that $A_{p}(\eta, \delta)=\bar{B}_{p}(\delta) \backslash B_{p}(\eta)$ ), then there exists a constant scalar curvature metric $\hat{g}$ on $M$ which coincides with $g$ in $M \backslash B_{p}(\delta)$, and which equals $\tilde{g}$ in $\bar{B}_{p}(\eta) \backslash$ $B_{p}(\eta / 2)$.

We are now in a position to prove Theorem 2.1. We first apply Lemma 2.2 and fix $r \in\left(0, r_{0}\right]$ such that

$$
\left\|g-g^{r}\right\|_{\mathcal{C}^{k}(A)} \leq \zeta / 2 .
$$

At this stage $r$ is fixed and we next apply Theorem 2.3 with $M_{0}=\bar{B}_{p}(\delta)$ and $g_{0}=g^{r}$ to obtain a family of asymptotically Delaunay metrics $\tilde{g}^{r, \epsilon}$, with neck size $\epsilon \in\left(0, \tilde{\epsilon}_{0}\right]$. Now, $g^{r, \epsilon}$ converges to $g^{r}$ in $\mathcal{C}^{k}(A)$ as $\epsilon$ tends to zero. Therefore, reducing $\epsilon_{0}$ if necessary, we can assume that

$$
\left\|g^{r, \epsilon}-g\right\|_{\mathcal{C}^{k}(A)} \leq \zeta
$$

and [4, Theorem 5.9 and Corollary 5.11] apply to produce a smooth constant scalar curvature metric $\stackrel{\circ}{g}^{r, \epsilon}$ which coincides with $g$ away from $B_{p}(\delta)$, and coincides with $g^{r, \epsilon}$ on $B_{p}(\eta)$. Observe that this metric is asymptotic to a Delaunay metric as explained in Theorem 2.3. Finally, applying Theorem 3.1 of [5], the metric $\stackrel{\circ}{g}^{r, \epsilon}$ can be deformed to a constant scalar curvature metric which is exactly Delaunay in the asymptotically Delaunay region. This completes the proof of our result.

\section{Acknowledgements}

The authors would like to warmly thank the referee for useful comments.

\section{References}

[1] R. Beig, P.T. Chruściel, and R. Schoen, KIDs are non-generic, Ann. H. Poincaré 6 (2005), 155-194, arXiv:gr-qc/0403042.

[2] A. Byde, Gluing theorems for constant scalar curvature manifolds, Indiana Univ. Math. Jour. 52 (2003), 1147-1199.

[3] L.A. Caffarelli, B. Gidas, and J. Spruck, Asymptotic symmetry and local behavior of semilinear elliptic equations with critical Sobolev growth, Commun. Pure Appl. Math. 42 (1989), 271-297. 
[4] P.T. Chruściel and E. Delay, On mapping properties of the general relativistic constraints operator in weighted function spaces, with applications, Mém. Soc. Math. de France. 94 (2003), vi+103, arXiv:gr-qc/0301073v2.

[5] P.T. Chruściel and D. Pollack, Singular Yamabe metrics and initial data with exactly KottlerSchwarzschild-de Sitter ends, Ann. H. Poincaré 9 (2008), 639-654, arXiv:0710.3365 [gr-qc].

[6] N. Korevaar, R. Mazzeo, F. Pacard, and R. Schoen, Refined asymptotics for constant scalar curvature metrics with isolated singularities, Invent. Math. 135 (1999), 233-272.

[7] R. Mazzeo and F. Pacard, Constant scalar curvature metrics with isolated singularities, Duke Math. J. 99 (1999), 353-418.

[8] R. Mazzeo, D. Pollack, and K. Uhlenbeck, Moduli spaces of singular Yamabe metrics, Jour. Amer. Math. Soc. 9 (1996), 303-344.

[9] A.G. Riess et al., New Hubble Space Telescope discoveries of type Ia Supernovae at $z>1$ : Narrowing constraints on the early behavior of dark energy, (2006), arXiv:astro-ph/0611572.

[10] W.M. Wood-Vasey et al., Observational constraints on the nature of the dark energy: First cosmological results from the essence supernova survey, (2007), arXiv:astro-ph/0701041.

LMPt, Fédération Denis Poisson, Tours, Mathematical Institute and Hertford ColLEGE, OXFORD

E-mail address: chrusciel@maths.ox.ac.uk

Université Paris Est and Institut Universitaire de France, Laboratoire d'Analyse et de Mathématiques Appliquées,, 61 Avenue du Général de Gaulle, 94010, Créteil, France

E-mail address: pacard@univ-paris12.fr

University of Washington, Mathematics Department, Box 354350 Seattle, WA 981954350, USA

E-mail address: pollack@math.washington.edu 